\title{
Angle Estimation and Mutual Coupling Self-Calibration in Bistatic MIMO System with Arbitrary Geometry
}

\author{
Tingping Zhang $\mathbb{D}^{1},{ }^{1}$ Di Wan $\mathbb{D}^{1},{ }^{1}$ Xinhai Wang $\mathbb{D}^{2},{ }^{2}$ and Fangqing Wen $\mathbb{D}^{3}$ \\ ${ }^{1}$ School of Information Science and Engineering, Chongqing Jiaotong University, Chongqing 400074, China \\ ${ }^{2}$ Nanjing Marine Radar Institute, Nanjing 211153, China \\ ${ }^{3}$ College of Computer and Information Technology, China Three Gorges University, Yichang 443002, China
}

Correspondence should be addressed to Tingping Zhang; ztp@cqjtu.edu.cn

Received 18 March 2021; Accepted 24 May 2021; Published 26 July 2021

Academic Editor: Guimei Zheng

Copyright (C) 2021 Tingping Zhang et al. This is an open access article distributed under the Creative Commons Attribution License, which permits unrestricted use, distribution, and reproduction in any medium, provided the original work is properly cited.

Ideal array responses are often desirable to a multiple-input multiple-output (MIMO) system. Unfortunately, it may not be guaranteed in practice as the mutual coupling (MC) effects always exist. Current works concerning MC in the MIMO system only account for the uniform array geometry scenario. In this paper, we generalize the issue of angle estimation and MC selfcalibration in a bistatic MIMO system in the case of arbitrary sensor geometry. The MC effects corresponding to the transmit array and the receive array are modeled by two MC matrices with several distinct entities. Angle estimation is then recast to a linear constrained quadratic problem. Inspired by the MC transformation property, a multiple signal classification- (MUSIC-) like strategy is proposed, which can estimate the direction-of-departure (DOD) and direction-of-arrival (DOA) via two individual spectrum searches. Thereafter, the MC coefficients are obtained by exploiting the orthogonality between the signal subspace and the noise subspace. The proposed method is suitable for arbitrary sensor geometry. Detailed analyses with respect to computational complexity, identifiability, and Cramer-Rao bounds (CRBs) are provided. Simulation results validate the effectiveness of the proposed method.

\section{Introduction}

Multiple-input multiple-output (MIMO) is the technique with the most potential for the next-generation array radar system [1-3]. A MIMO system is characterized by multiple transmitting antennas and multiple receiving antennas. Unlike the traditional phased array radar, the MIMO system emits mutual orthogonal waveforms. The spatial diversity and waveform diversity enables the MIMO system to achieve a virtual aperture, which is much larger than the physical aperture of the radar system. Benefiting from the virtual aperture, the MIMO system outperforms the phased array radar with respect to clutter suppression, direction finding, and target tracking. Usually, the MIMO system can be divided into two categories in terms of its antenna configuration, namely, a distributed MIMO system and a colocated MIMO system [4, 5]. A distributed MIMO system is equipped with widely displaced transmitting and receiving $(\mathrm{Tx} / \mathrm{Rx})$ antennas, and it can illuminate the area of interest from various perspectives. Therefore, it is capable of extinguishing the radar cross-section (RCS) fluctuation issue. A colocated MIMO system consists of closely spaced Tx/Rx antennas, and it is able to provide super-resolution direction estimation. Herein, we focus on the bistatic MIMO system, which is a typical representative of the colocated MIMO system.

As a canonical issue in bistatic MIMO systems, joint direction-of-departure (DOD) and direction-of-arrival (DOA) estimation has been studied extensively in the past decades. Various methodologies have been proposed, e.g., estimation method of signal parameters via rotational invariance technique (ESPRIT) [6], multiple signal classification (MUSIC) [7], maximum likelihood [8], tensor approaches $[9,10]$, and sparsity-aware methods $[11,12]$. A common assumption in [6-11] is that the sensors are well-calibrated. Nevertheless, owing to the radiation effects [13], sensors may be susceptible to unknown mutual coupling (MC), which would bring perturbation to the signal model and thus 
lead to degraded estimation performance. In general, the MC effect between sensors can be described by a MC matrix, in which the entities reveal the strength between two sensors. Although the MC matrix can be directly obtained offline via the measurement techniques such as the electromotive force [14], it may be invalid in the scenario where $\mathrm{MC}$ varies with time. To pursue online MC calibration, the usage of instrumental sensors or auxiliary sources have been reported $[15,16]$, which require additional resource. As is known to us, the MC intensity of two sensors is inversely proportional to their distance. Therefore, the MC effects between sensors that are far apart can be neglected, i.e., the associated MC coefficients can be approximated by zeros. As a result, sparse arrays, e.g., coprime arrays $[17,18]$, may be free from MC, but it is impossible for applications with limited space.

As mentioned earlier, the MC intensity between two sensors is inversely proportional to their interelement distance. Accordingly, the MC matrix associated with a uniform linear array (ULA) exhibits a special structure, which can be captured by a banded symmetric Toeplitz matrix. For a ULAbased MIMO system, several strategies have been proposed for MC self-calibration. In [19], a MUSIC-like estimator was presented, in which the MC coefficients are extracted into a single vector, and the DODs and DOAs are obtained via two one-dimensional (1D) peak searches. By interpreting the sensors at both ends of the arrays as instrumental sensors, the ESPRIT-like algorithms were introduced in $[20,21]$, where the MC effect is suppressed by using a selection matrix, and then the ESPRIT technique is followed to obtain closeform solutions for DOD and DOA estimation. In order to exploit the tensor structure of the array data, the tensor approaches were subsequently discussed in [22-24], which offer more accurate angle estimation performance than the matrix-based ones. Besides, some frameworks have investigated into the two-dimensional (2D) angle estimation issue using a polarization-sensitive array [25-28].

It should be emphasized that the above algorithms are only effective for MIMO systems with ULA configurations. In practice, the ULA-based MIMO system may occasionally encounter sensor failure [29, 30]. Besides, a more complex array manifold would be adopted in the MIMO system [31]. Therefore, the MIMO system with arbitrary $\mathrm{Tx} / \mathrm{Rx}$ geometries may suffer from unknown MC, and current estimators in [1924 ] will fail to work. To the best of our knowledge, only a few efforts have been devoted to robust DOA estimation for arbitrary geometry sensor array with unknown MC. In such a case, the MC matrix exhibits no special structure except Hermitian. In [32], an optimization-based framework was investigated. It formulates the DOA estimation as a block sparse inverse problem, which is solved via a joint-sparse recovery algorithm. Like the traditional counterpart, it has high complexity due to the high-dimension nonconvex problem, making it unacceptable for the MIMO system. In [33], an iterative approach was considered, in which DOA and MC coefficients are updated alternately. More recently, the MUSIC-like method was applied to robust DOA estimation in [34]. Although the approaches in $[33,34]$ can be directly extended to angle estimation for a bistatic MIMO system, they are inefficient due to an exhaustive grid search.
In this paper, we generalize the $\mathrm{MC}$ issue in a bistatic MIMO system. We consider a general scenario where the $\mathrm{Tx} / \mathrm{Rx}$ arrays are in arbitrary geometries and MC effects occur in both the $\mathrm{Tx} / \mathrm{Rx}$ arrays. In such a scenario, the $\mathrm{MC}$ matrices exhibit no special structure except symmetry. To tackle the DOD and DOA estimation problem, the eigendecomposition is firstly performed to obtain the noise subspace. Then, by exploiting the orthogonality between the noise subspace and the virtual steering vector, angle estimation is recast to a general quadratic problem. Therefore, the $\mathrm{MC}$ transformation property is adopted to transform the optimization problem into two different forms, in which only DOD and DOA are included. Consequently, DOD and DOA can be estimated via two individual spectrum searches. Finally, the MC coefficients can be easily obtained with the estimated DOD and DOA. The proposed method is analyzed in terms of computational complexity, identifiability, and CramerRao bounds (CRBs). Numerical simulations are designed to show the effectiveness of the proposed method.

Notation, bold capital letters, e.g., $\mathbf{X}$, and bold lowercase letters, e.g., $\mathbf{x}$, denote matrices and vectors, respectively. The superscripts $(\cdot)^{\mathrm{T}},(\cdot)^{\mathrm{H}}$, and $(\cdot)^{\dagger}$ account for transpose, Hermitian transpose, and inverse, respectively. The identity matrix is denoted by $\mathbf{I}$, the $M \times N$ full one matrix is denoted by $1_{M \times N}$, the full zero matrix is denoted by $0 . \otimes, \odot$, and $\oplus$ represent, respectively, the Kronecker product, the KhatriRao product, and the Hadamard product; diag $\{\cdot\}$ denotes the diagonalization operation; $\operatorname{rank}(\cdot)$ denotes rank operator; and $\operatorname{Re}(\cdot)$ and $\operatorname{Im}(\cdot)$ return the real part and the image part of a vector, respectively.

\section{Problem Formulation}

We consider a bistatic MIMO system with $M$ transmit sensors and $N$ receive sensors, and there are $K$ far-field targets appearing in the same range bins of the radar system. The 2D-DOA pair and the 2D-DOA of the $k$ th $(k=1,2, \cdots, K)$ target are denoted by $\Theta_{t, k}=\left[\theta_{t, k}, \phi_{t, k}\right]^{\mathrm{T}}$ and $\Theta_{r, k}=\left[\theta_{t, k}, \phi_{t, k}\right]^{\mathrm{T}}$, where $\theta_{t, k}$ and $\theta_{r, k}$ are elevation angles and $\phi_{t, k}$ and $\phi_{r, k}$ are azimuth angles. Without loss of generality, we assume that both the transmit sensors and the receive sensors are distributed in arbitrary geometries. The coordinate of the $m$ th $(m$ $=1,2, \cdots, M)$ transmit sensor is $p_{t, m}=\left[x_{t, m}, y_{t, m}, z_{t, m}\right]^{\mathrm{T}}$, and the coordinate of the $n$th $(n=1,2, \cdots, M)$ receive sensor is $p_{r, n}=\left[x_{r, n}, y_{r, n}, z_{r, n}\right]^{\mathrm{T}}$. The response vectors corresponding to the transmit array and the receive array are given by

$$
a\left(\Theta_{t, k}\right)=\left[\exp \left\{-\frac{j 2 \pi \tau_{1, k}}{\lambda}\right\}, \exp \left\{-\frac{j 2 \pi \tau_{2, k}}{\lambda}\right\}, \cdots, \exp \left\{-\frac{j 2 \pi \tau_{M, K}}{\lambda}\right\}\right]^{\mathrm{T}},
$$

$$
a\left(\Theta_{r, k}\right)=\left[\exp \left\{-\frac{j 2 \pi \Delta_{1, K}}{\lambda}\right\}, \exp \left\{-\frac{j 2 \pi \Delta_{2, K}}{\lambda}\right\}, \cdots, \exp \left\{-\frac{j 2 \pi \Delta_{N, K}}{\lambda}\right\}\right]^{\mathrm{T}},
$$

where $\lambda$ is the carrier wavelength, $\tau_{m, k}$ and $\Delta_{n, k}$ are the associated path differences with $\tau_{m, k}=\mathbf{p}_{t, m}^{T} \mathbf{f}_{t, k}, \Delta_{n, k}=\mathbf{p}_{r, n}^{T} \mathbf{f}_{r, k}, \mathbf{f}_{t, k}=$ 
$\left[\cos \left(\phi_{t, k}\right) \sin \left(\theta_{t, k}\right), \sin \left(\phi_{t, k}\right) \sin \left(\theta_{t, k}\right), \cos \left(\theta_{t, k}\right)\right]^{\mathrm{T}}$, and $f_{r, k}$ $=\left[\cos \left(\phi_{r, k}\right) \sin \left(\theta_{r, k}\right), \sin \left(\phi_{r, k}\right) \sin \left(\theta_{r, k}\right), \cos \left(\theta_{r, k}\right)\right]^{\mathrm{T}}$,

respectively. Define $\mathbf{A}_{t}=\left[\mathbf{a}\left(\Theta_{t, 1}\right), \mathbf{a}\left(\Theta_{t, 2}\right), \cdots, \mathbf{a}\left(\Theta_{t, k}\right)\right] \in C^{M \times K}$ and $\operatorname{Ar}=\left[\mathrm{a}\left(\Theta_{r, 1}\right), \mathrm{a}\left(\Theta_{r, 2}\right), \cdots, \mathrm{a}\left(\Theta_{r, k}\right)\right] \in C^{N \times K}$ are the direction matrices corresponding to the transmit array and receive array, respectively. In the absence of the MC effect, the output from the matched filters can be written as

$x(t)=\sum_{k=1}^{k}\left[\mathrm{a}\left(\Theta_{r, k}\right) \otimes \mathrm{a}\left(\boldsymbol{\Theta}_{t, k}\right)\right] s_{k}(t)+\mathrm{N}(t)=\left[\mathrm{A}_{r} \odot \mathrm{A}_{t}\right] s(t)+\mathbf{n}(t)$,

where $t$ is the slow time index; $s_{k}(t)$ is the RCS coefficient of the $k$ th targets; $\mathbf{n}(t)$ is the array noise, which is assumed to be Gaussian white with variance $\sigma^{2}$; and $s(t)=$ $\left[s_{1}(t), s_{2}(t), \cdots, s_{k}(t)\right]^{\mathrm{T}}$. Now, we consider a scenario where both the transit sensors and the receive sensors suffer from unknown MC and the MC matrices are denoted by $\mathbf{C}_{t} \in$ $C^{M \times M}$ and $\mathbf{C}_{r} \in C^{N \times N}$, respectively, which are Hermitian. In the presence of an unknown MC, the matched output in (2) becomes

$$
\begin{aligned}
y(t) & =\sum_{K=1}^{K}\left[\left(C_{r} a\left(\Theta_{r, k}\right)\right) \otimes\left(C_{r} a\left(\Theta_{t, k}\right)\right)\right] s_{k}(t)+n(t) \\
& =\underbrace{\left[\left(\mathbf{C}_{r} \mathbf{A}_{r}\right) \odot\left(\mathbf{C}_{t} \mathbf{A}_{t}\right)\right]}_{\mathbf{A}} \mathbf{s}(t)+\mathbf{n}(t) .
\end{aligned}
$$

Suppose the RCS coefficients are uncorrelated, and $s(t)$ is irrelevant with $n(t)$, and then, the covariance matrix of $y(t)$ is

$$
\mathbf{R}=\mathbf{A} \mathbf{R}_{s} \mathbf{A}^{H}+\sigma^{2} \mathbf{I},
$$

where $\mathbf{R}_{s}=\operatorname{diag}\left\{\gamma_{1}, \gamma_{2}, \cdots, \gamma_{k}\right\}$ is the covariance matrix of $s(t)$ , $\gamma_{k}$ is the power of $s_{k}(t)$. With $L$ available snapshots, i.e., $t$ $=1,2, \cdots, L, R$ can be estimated via

$$
\widehat{\mathrm{R}}=\frac{1}{L} \sum_{t=1}^{L} \mathrm{y}(t) \mathrm{y}^{H}(t)
$$

\section{The Proposed Algorithm}

3.1. DOD and DOA Estimation. Performing eigendecomposition on $\mathbf{R}$, one can obtain the signal subspace and the noise subspace as

$$
\mathbf{R}=\left[\mathbf{U}_{s}, \mathbf{U}_{n}\right]\left[\begin{array}{ll}
\Lambda_{s} & \\
& \Lambda_{n}
\end{array}\right]\left[\begin{array}{c}
\mathbf{U}_{s}^{H} \\
\mathbf{U}_{n}^{H}
\end{array}\right]
$$

where $\Lambda_{s} \in C^{K \times K}$ and $\Lambda_{n} \in C^{(M N-K) \times(M N-K)}$ are two diagonal matrices that contain the $K$ dominate eigenvalues and the remind eigenvalues, respectively. $\mathbf{U}_{s} \in C^{M N \times K}$ and $\mathbf{U}_{n} \in$ $C^{M N \times(M N-K)}$ consist of the corresponding eigenvectors, which are called the signal subspace and the noise subspace, respectively. It is known that $\mathbf{U}_{s}$ is orthogonal to $\mathbf{U}_{n}$, i.e.,

$$
\mathbf{U}_{n}^{H} \mathbf{U}_{s}=0
$$

Since $\mathbf{U}_{s}$ span the same subspace with the columns of $\mathbf{A}$, we get

$$
\left[\left(\mathbf{C}_{r} \mathbf{a}\left(\Theta_{r, k}\right)\right) \otimes\left(\mathbf{C}_{t} \mathbf{a}\left(\Theta_{t, k}\right)\right)\right]^{H} \mathbf{U}_{n} \mathbf{U}_{n}^{H}\left[\left(\mathbf{C}_{r} \mathbf{a}\left(\Theta_{r, k}\right)\right) \otimes\left(\mathbf{C}_{t} \mathbf{a}\left(\Theta_{t, k}\right)\right)\right]=0
$$

The above property is similar to that in the 2D-MUSIC algorithm. However, the traditional MUSIC estimator is invalid as both $\mathbf{C}_{t}$ and $\mathbf{C}_{r}$ are unknown. To further process, the following conclusion in [33] is introduced.

Theorem 1. For a matrix $\mathbf{C} \in C^{M \times M}$ and a vector $\mathbf{a} \in C^{M \times 1}$, if there are only $Q(Q<M)$ distinct entities $\mathbf{c}=\left[c 1, c 2, \cdots, c_{Q}\right]^{T}$ in $C$, then

$$
C a=T c,
$$

where $\mathbf{T} \in C^{M \times Q}$ with the qth $(q=1,2, \cdots, Q)$ column given by

$$
\mathbf{T}(:, q)=\mathbf{J}_{q} \mathbf{a}
$$

and $\boldsymbol{J}_{q}$ is defined as

$$
\mathbf{J}_{q}(m, n)= \begin{cases}1, & \text { if } \mathbf{C}(m, n)=c_{q} \\ 0, & \text { otherwise. }\end{cases}
$$

In this paper, we build on top of Theorem 1 to address the MC self-calibration challenge in the arbitrary geometrybased MIMO system. Suppose that there are $P$ distinct entities $\mathbf{c}_{t}=\left[c_{t, 1}, c_{t, 2}, \cdots, c_{t, p}\right]^{\mathrm{T}}$ in $\mathbf{C}_{t}$, and $Q$ distinct entities $\mathbf{c}_{r}=$ $\left[c_{r, 1}, c_{r, 2}, \cdots, c_{r, Q}\right]^{\mathrm{T}}$ in $\mathbf{C}_{r}$. From Theorem 1, we have

$$
\begin{gathered}
\mathbf{C}_{t} \mathbf{a}\left(\Theta_{t, k}\right)=\mathbf{T}\left(\Theta_{t, k}\right) \mathbf{c}_{t}, \\
\mathbf{C}_{r} \mathbf{a}\left(\Theta_{r, k}\right)=\mathbf{T}\left(\Theta_{r, k}\right) \mathbf{c}_{r},
\end{gathered}
$$

where $\mathbf{T}\left(\Theta_{t}\right) \in C^{\mathrm{M} \times \mathrm{P}}$ and $\mathbf{T}\left(\Theta_{r}\right) \in C^{\mathrm{N} \times \mathrm{Q}}$ are constructed according to Theorem 1 . In combination with the property $(A \otimes B)(C \otimes D)=(A C) \otimes(B D)$, we can obtain

$$
\begin{aligned}
\left(C_{r} \mathrm{a}\left(\Theta_{r, k}\right)\right) \otimes\left(C_{t} \mathrm{a}\left(\Theta_{t, k}\right)\right) & =\underbrace{\left(T_{r}\left(\Theta_{r, k}\right)\right.}_{A} c_{B}^{\left.c_{r}\right)} \otimes \underbrace{I_{M}}_{C} \underbrace{C_{t} \mathrm{a}\left(\Theta_{t, k}\right)}_{D} \\
& =\left(T\left(\Theta_{r, k}\right) \otimes I_{M}\right)\left(c_{r} \otimes\left[C_{t} \mathrm{a}\left(\Theta_{t, k}\right)\right]\right),
\end{aligned}
$$

$$
\begin{aligned}
\left(C_{r} \mathrm{a}\left(\Theta_{r, k}\right)\right) \otimes\left(C_{t} \mathrm{a}\left(\Theta_{t, k}\right)\right) & =\underbrace{\left(T_{r}\left(\Theta_{r, k}\right)\right.}_{A} c_{B}^{\left.c_{r}\right)} \otimes \underbrace{I_{M}}_{C} \underbrace{C_{t} \mathrm{a}\left(\Theta_{t, k}\right)}_{D} \\
& =\left(I_{N} \otimes T\left(\Theta_{t, k}\right)\right)\left(\left[C_{r} \mathrm{a}\left(\Theta_{r, k}\right)\right] \otimes c_{t}\right) .
\end{aligned}
$$


Inserting (13a) and (13b) into (9) establishes

$$
e^{H}\left(\Theta_{t, k}\right) \underbrace{\left[\left(T\left(\Theta_{r, k}\right) \otimes I_{M}\right)\right]^{H} U_{n} U_{n}^{H}\left[\left(T\left(\Theta_{r, k}\right) \otimes I_{M}\right)\right]}_{P\left(\Theta_{r, k}\right)} \mathrm{e}\left(\Theta_{t, k}\right)=0,
$$

$$
f^{H}\left(\Theta_{r, k}\right) \underbrace{\left[\left(I_{N} \otimes T\left(\Theta_{t, k}\right)\right)\right]^{H} U_{n} U_{n}^{H}\left[\left(I_{N} \otimes T\left(\Theta_{t, k}\right)\right)\right]}_{Q\left(\Theta_{t, k}\right)} \mathrm{f}\left(\Theta_{r, k}\right)=0,
$$

where $\mathbf{e}\left(\Theta_{t, k}\right)=c_{t} \otimes\left[\mathbf{C}_{t} \mathbf{a}\left(\Theta_{t, k}\right)\right]$ and $\mathbf{f}\left(\Theta_{r, k}\right)=\left[\mathbf{C}_{r} \mathbf{a}\left(\Theta_{r, k}\right)\right] \otimes \mathbf{c}_{r}$. To obtain the DOA, one needs to optimize

$$
\min \quad e^{H} P(\Theta) e \quad \text { s.t., } \quad d^{H} e=\rho .
$$

Notably, (15) is a quadratic optimization issue. To avoid the trivial solution $\mathbf{e}=0_{M P \times 1}$, the above optimization problem is constrained with $d^{H} e=\rho$, i.e.,

$$
\min \quad e^{H} \mathrm{P}(\Theta) e \quad \text { s.t., } \quad \mathrm{d}^{H} e=\rho,
$$

where $\rho$ is a constant and $\mathbf{d}$ is a vector with the first entity being one and zeros elsewhere. To solve the above issue, we can construct the following Lagrange function:

$$
\mathscr{L}(\Theta)=e^{H} p(\Theta) e-\mathscr{T}\left(\frac{d^{H} e}{\rho}-1\right)
$$

where $\mathscr{T}$ is a Lagrange multiplier. Enforcing $\partial \mathscr{L}(\Theta) / c$ to zeros yields

$$
e=\frac{\rho^{2} \mathrm{P}^{-1}(\Theta) \mathrm{d}}{d^{H} P^{-1}(\Theta) \mathrm{d}}
$$

Plug (18) into (15) and remove the constant item gives

$$
\max d^{H} P^{-1}(\Theta) \mathrm{d}
$$

By setting a grid to contain all possible DODs, the indexes corresponding to the maximum values in (19) reveal the DODs. Similarly, we can obtain the DOA estimation via computing

$$
\max g^{H} Q^{-1}(\Theta) g
$$

where $\mathbf{g}$ is a NQ $\times 1$ vector with the first entity one and zeros elsewhere.

3.2. MC Self-Calibration. Although DOD $\Theta_{t, k}$ and DOA $\Theta_{r, k}$ can be separately estimated from (19) and (20), they require further pairing, since there are $K(K-1) / 2$ possible DODDOA pairs. Recall the properties in (12) and we can rewrite (8) as

$$
c_{t r}^{H} \underbrace{\left[T\left(\Theta_{r, k}\right) \otimes T\left(\Theta_{t, k}\right)\right]^{H} U_{n} U_{n}^{H}\left[\mathrm{~T}\left(\Theta_{r, k}\right) \otimes \mathrm{T}\left(\Theta_{t, k}\right)\right]}_{T\left(\Theta_{t, k}, \Theta_{r, k}\right)} c_{t r}=0,
$$

where $c_{t r}=c_{r} \otimes c_{t}$. Similar to (15), to find the correct DODDOA pairs, we need to calculate

$$
\min c_{t r}^{H} \mathrm{~T}\left(\Theta_{t}, \Theta_{r}\right) c_{t r}
$$

which can be accomplished via

$$
\max h^{H} T^{-1}\left(\Theta_{t}, \Theta_{r}\right) \mathrm{h},
$$

where $h$ is a $\mathrm{PQ} \times 1$ vector with the first entity one and zeros elsewhere. List all the possible DOD-DOA pairs, and the real DOD-DOA pairs can be picked out by finding the $K$ maximum values in (23).

It should be pointed out that the optimal solution of (22) is achieved if

$$
\mathbf{c}_{t r}=\frac{\alpha^{2} \mathbf{T}^{-1}\left(\Theta_{t}, \Theta_{r}\right) \mathbf{h}}{\mathbf{h}^{H} \mathbf{T}^{-1}\left(\Theta_{t}, \Theta_{r}\right) \mathbf{h}}
$$

where $\alpha$ is a constant. After the DODs and DOAs have been paired, $c_{t r}$ can be estimated via

$$
\widehat{\mathbf{c}}_{t, r}=\frac{1}{K} \sum_{k=1}^{K} \frac{\alpha^{2} \mathbf{T}^{-1}\left(\widehat{\Theta}_{t, k}, \widehat{\Theta}_{r, k}\right) \mathbf{h}}{\mathbf{h}^{H}\left(\widehat{\Theta}_{t, k}, \widehat{\Theta}_{r, k}\right) \mathbf{h}},
$$

where $\widehat{\Theta}_{t, k}$ and $\widehat{\Theta}_{r, k}$ denote the estimated $\Theta_{t, k}$ and $\Theta_{r, k}$, respectively. Usually, we have $c_{t, 1}=1$ and $c_{r, 1}=1$. After normalizing $\widehat{\mathbf{c}}_{t, r}$, the $\mathrm{MC}$ vectors can be estimated via

$$
\widehat{\mathbf{c}}_{t}=\mathbf{J}_{M} \widehat{\mathbf{c}}_{t, r}
$$

$$
\widehat{\mathbf{c}}_{r}=\mathbf{J}_{N} \widehat{\mathbf{c}}_{t, r},
$$

where $\mathbf{J}_{M}=[1,0] \otimes \mathbf{I} \in \mathbb{R}^{M \times M N}, \mathbf{J}_{N}=\mathbf{I} \otimes\left[1,0_{(M-1) \times 1}^{T}\right] \in \mathbb{R}^{N \times M N}$.

\section{Algorithm Analysis}

4.1. Computational Complexity. The computational burden (the number of complex multiplication) of the proposed is summarized as follows:

Step 1. Estimate the covariance matrix $\widehat{\mathbf{R}} \cdots \cdots \cdots M^{2} N^{2} L$.

Step 2. Perform eigendecomposition on $\widehat{\mathbf{R}}$ to obtain the noise subspace $U_{n} \cdots \cdots \cdot O\left\{M^{3} N^{3}\right\}$.

Step 3. Calculate the spectrum function in (19) to get the DOD estimation ….... $O\left\{M^{3} N^{2} Q\right\}$. 
Step 4. Search the maximums of (20) to achieve the DOA estimation ….... $\left\{M^{2} N^{3} P\right\}$.

4.2. Identifiability. The proposed algorithm is effective if $P^{-1}(\Theta), Q^{-1}(\Theta)$, and $T^{-1}\left(\Theta_{\mathbf{t}}, \Theta_{\mathbf{r}}\right)$ are valid. As rank $\left\{U_{n}\right.$ $\left.U_{n}^{H}\right\}=M N-K$, once $M Q>M N-K, P(\Theta)$ is rank deficit. Similarity, if $N Q>M N-K, \mathbf{Q}(\Theta)$ is nonfull rank, and if $P$ $Q>M N-K$, rank deficiency would occur in $T\left(\Theta_{\mathbf{t}}, \Theta_{\mathbf{r}}\right)$. As a result, a sufficient condition of the proposed estimator is that $K \leq \min \{M(N-P), N(M-Q), M N-P Q\}$. Since $M>P$ and $N>Q$, the above condition can be simplified by $K \leq \min \{M(N-P), N(M-Q)\}$. In other words, the proposed estimator can identify $\min \{M(N-P), N(M-Q)\}$ targets at most.

4.3. Cramer-Rao Bound (CRB). For the sake of simplicity, (3) is rewritten as

$$
y_{t}=A s_{t}+n_{t}, \quad t=1,2, \cdots, L,
$$

where $\mathbf{y}_{t} \triangleq \mathbf{y}(t), \mathbf{s}_{t}=\mathbf{s}(t)$, and $\mathbf{n}_{t}=\mathbf{n}(t)$. Then, all the measurements are arranged into a "column" vector as $\mathbf{y}=$ $\left[\mathbf{y}_{t}^{T}, \mathbf{y}_{2}^{T} \cdots, y_{L}^{T}\right]^{T} \in C^{M N L \times 1}$. Suppose that the $\mathbf{s}_{t}$ is deterministic but unknown. Thereafter, the mean $\mu \in C^{\mathrm{MNL} \times 1}$ of $\mathbf{y}$ is given by

$$
\mu=\left[\begin{array}{c}
\mathrm{As}_{1} \\
\mathrm{As}_{2} \\
\vdots \\
\mathrm{As}_{L}
\end{array}\right]=\mathrm{HF}
$$

where $\mathbf{H} \triangleq \mathbf{I} \otimes \boldsymbol{A} \in \mathbb{C}^{M N L \times L K}$ and $\mathbf{F} \triangleq\left[\mathbf{s}_{1}^{T}, \mathbf{s}_{2}^{T} \cdots \mathbf{s}_{L}^{T}\right]^{T} \in \mathbb{C}^{L K \times 1}$.

Define the unknown parameter vectors $\theta_{t} \triangleq$ $\left[\theta_{t, 1}, \theta_{t, 2}, \cdots, \theta_{t, k}\right]^{\mathrm{T}}, \phi_{t} \triangleq\left[\phi_{t, 1}, \phi_{t, 1} \cdots, \phi_{t, 1}\right]^{\mathrm{T}}, \theta r,\left[\theta_{t, 1}, \theta_{r, 1}, \cdots, \theta_{r, k}\right]^{\mathrm{T}}$ $, \quad \phi_{r} \triangleq\left[\phi_{r, 1}, \phi_{r, 2} \cdots, \phi_{r, k}\right]^{\mathrm{T}}, \quad \alpha \triangleq\left[\theta_{t}^{T}, \theta_{r}^{T}, \phi_{t}^{T}, \phi_{r}^{T}\right]^{T} \in R^{4 K \times 1}, \quad \beta \triangleq$ $\left[\operatorname{Re}\left\{c_{r}^{T}\right\}, \operatorname{Re}\left\{c_{t}^{T}\right\}, \operatorname{Im}\left\{c_{r}^{T}\right\}, \operatorname{Im}\left\{c_{t}^{T}\right\}\right]^{T} \in R^{2(P+Q) \times 1}$, and $\gamma \triangleq$ $\left[\operatorname{Re}\left\{\mathbf{F}^{\mathrm{T}}\right\}, \operatorname{Im}\left\{\mathbf{F}^{\mathrm{T}}\right\}\right]^{\mathrm{T}} \in R^{2 L K \times 1}$. The whole estimation parameter vector is formulated as $\zeta \triangleq\left[\alpha^{\mathrm{T}}, \beta^{\mathrm{T}}, \gamma^{\mathrm{T}}\right]^{\mathrm{T}} \in R^{2(L K+2 K+P+Q) \times 1}$. It is well known that the CRB matrix for $\zeta$ is given by

$$
\mathrm{CRB}=\frac{\sigma^{2}}{2}\left[\operatorname{Re}\left\{\Psi^{H} \Psi\right\}\right]^{-1},
$$

where $\Psi \triangleq\left[\partial \mu / \partial \alpha^{T}, \partial \mu / \partial \beta^{T}, \partial \mu / \partial \gamma^{T}\right]$. Next, we will concentrate on each part of $\Psi$. Before the detailed derivations, the following variables are defined:

$$
\begin{aligned}
& \tilde{\mathbf{A}}_{t, 1} \triangleq\left[\frac{\partial \mathbf{a}\left(\Theta_{t, 1}\right)}{\partial \theta_{t, 1}}, \frac{\partial \mathbf{a}\left(\Theta_{t, 2}\right)}{\partial \theta_{t, 2}}, \cdots, \frac{\partial \mathbf{a}\left(\Theta_{t, K}\right)}{\partial \theta_{t, K}}\right], \\
& \tilde{\mathbf{A}}_{t, 2} \triangleq\left[\frac{\partial \mathbf{a}\left(\Theta_{t, 1}\right)}{\partial \phi_{t, 1}}, \frac{\partial \mathbf{a}\left(\Theta_{t, 2}\right)}{\partial \phi_{t, 2}}, \cdots, \frac{\partial \mathbf{a}\left(\Theta_{t, K}\right)}{\partial \phi_{t, K}}\right],
\end{aligned}
$$

$$
\begin{aligned}
& \tilde{\mathbf{A}}_{r, 1} \triangleq\left[\frac{\partial \mathbf{a}\left(\Theta_{r, 1}\right)}{\partial \theta_{r, 1}}, \frac{\partial \mathbf{a}\left(\Theta_{r, 2}\right)}{\partial \theta_{r, 2}}, \cdots, \frac{\partial \mathbf{a}\left(\Theta_{r, K}\right)}{\partial \theta_{r, K}}\right], \\
& \tilde{\mathbf{A}}_{r, 2} \triangleq\left[\frac{\partial \mathbf{a}\left(\Theta_{r, 1}\right)}{\partial \phi_{r, 1}}, \frac{\partial \mathbf{a}\left(\Theta_{r, 2}\right)}{\partial \phi_{r, 2}}, \cdots, \frac{\partial \mathbf{a}\left(\Theta_{r, K}\right)}{\partial \phi_{r, K}}\right], \\
& \tilde{\mathbf{C}}_{t, p} \triangleq\left[\frac{\partial \mathbf{C}_{t}}{\partial \operatorname{Re}\left\{c_{t, 1}\right\}}, \frac{\partial \mathbf{C}_{t}}{\partial \operatorname{Re}\left\{c_{t, 2}\right\}}, \cdots, \frac{\partial \mathbf{C}_{t}}{\partial \operatorname{Re}\left\{c_{t, P}\right\}}\right], \\
& \tilde{\mathbf{C}}_{r, q} \triangleq\left[\frac{\partial \mathbf{C}_{r}}{\partial \operatorname{Re}\left\{c_{r, 1}\right\}}, \frac{\partial \mathbf{C}_{r}}{\partial \operatorname{Re}\left\{c_{r, 2}\right\}}, \cdots, \frac{\partial \mathbf{C}_{r}}{\partial \operatorname{Re}\left\{c_{r, Q}\right\}}\right], \\
& \tilde{\mathbf{A}}_{1} \triangleq\left(\mathrm{C}_{r} \mathbf{A}_{r}\right) \odot\left(\mathrm{C}_{t} \tilde{\mathbf{A}}_{t, 1}\right), \\
& \tilde{\mathbf{A}}_{2} \triangleq\left(\mathrm{C}_{r} \mathbf{A}_{r}\right) \odot\left(\mathrm{C}_{t} \tilde{\mathbf{A}}_{t, 2}\right), \\
& \tilde{\mathbf{A}}_{3} \triangleq\left(\mathrm{C}_{r} \tilde{\mathbf{A}}_{r, 1}\right) \odot\left(\mathrm{C}_{t} \mathbf{A}_{t}\right) \\
& \tilde{\mathbf{A}}_{4} \triangleq\left(\mathrm{C}_{r} \tilde{\mathbf{A}}_{r, 2}\right) \odot\left(\mathrm{C}_{t} \mathbf{A}_{t}\right), \\
& \tilde{\mathbf{A}}_{t, p} \triangleq\left(\mathbf{C}_{r} \mathbf{A}\right) \odot\left(\tilde{\mathbf{C}}_{t, p} \mathbf{A}_{t}\right), \\
& \tilde{\mathbf{A}}_{r, q} \triangleq\left(\tilde{\mathbf{C}}_{r, q} \mathbf{A}_{r}\right) \odot\left(\mathbf{C}_{t} \mathbf{A}_{t}\right), \\
& \mathbf{S} \triangleq\left[\mathbf{s}_{1}, \mathbf{s}_{2}, \cdots, \mathbf{s}_{L}\right]^{T}, \\
& \tilde{\mathbf{A}}=\left[\tilde{\mathbf{A}}_{1}, \tilde{\mathbf{A}}_{2}, \tilde{\mathbf{A}}_{3}, \tilde{\mathbf{A}}_{4}\right], \\
& \tilde{\mathbf{B}}=\left[\tilde{\mathbf{A}}_{t, 1}, \cdots, \tilde{\mathbf{A}}_{t, P}, \tilde{\mathbf{A}}_{r, 1}, \cdots, \tilde{\mathbf{A}}_{r, Q}\right] \otimes[1, j] .
\end{aligned}
$$

It is easy to find that $\partial \mu / \partial \theta_{T}^{T}=\mathbf{S} \odot \tilde{\mathbf{A}}_{1}$. Stepping further, we can get

$$
\Delta \triangleq \frac{\partial \mu}{\partial \alpha^{T}} \in \mathbb{C}^{M N L \times 4 K}=\left[1_{1 \times 4} \otimes \mathbf{S}\right] \odot \tilde{\mathbf{A}} .
$$

As $\partial \mu / \partial \operatorname{Re}\left\{c_{t, p}\right\}=\mathbf{S} \odot \tilde{\mathbf{A}}_{t, p}$, and $\partial \mu / \partial \operatorname{Im}\left\{c_{t, p}\right\}=j(\mathbf{S} \odot$ $\left.\tilde{\mathbf{A}}_{t, p}\right)$, thus, we have

$$
\nabla \triangleq \frac{\partial \mu}{\partial \beta^{T}} \in \mathbb{C}^{M N L \times 2(P+Q)}=\left[1_{1 \times 2(P+Q)} \otimes \mathbf{S}\right] \odot \tilde{\mathbf{B}} .
$$

Besides, it is straightforward to get

$$
\frac{\partial \mu}{\partial \gamma^{T}}=[\mathbf{H}, j \mathbf{H}] \in \mathbb{C}^{M N L \times 2 L K} .
$$

Consequently, it is established that

$$
J \triangleq \operatorname{Re}\left\{\Psi^{H} \Psi\right\}=\operatorname{Re}\left\{\left[\begin{array}{c}
\Delta^{H} \\
\nabla^{H} \\
\mathbf{H}^{H} \\
-j \mathbf{H}^{H}
\end{array}\right][\Delta, \nabla, \mathbf{H}, j \mathbf{H}]\right\} .
$$


Since we are interested in CRBs on angle and MC estimation only, we will remove the uninterested parts from J. Define

$$
\begin{array}{r}
\mathbf{P}_{\Delta} \triangleq\left(\mathbf{H}^{H} \mathbf{H}\right)^{-1} \mathbf{H}^{H} \Delta \in \mathbb{C}^{L K \times 4 K}, \\
\mathbf{P}_{\nabla} \triangleq\left(\mathbf{H}^{H} \mathbf{H}\right)^{-1} \mathbf{H}^{H} \nabla \in \mathbb{C}^{L K \times 2(P+Q)} .
\end{array}
$$

Both $\mathbf{P}_{\nabla}^{-1}$ and $\mathbf{P}_{\nabla}^{-1}$ are valid as $\mathbf{H}^{\mathrm{H}} \mathbf{H}$ is nonsingular. Furthermore, the following transform matrix is defined:

$$
\mathrm{V} \triangleq\left[\begin{array}{cccc}
\mathbf{I} & 0 & 0 & 0 \\
0 & \mathbf{I} & 0 & 0 \\
-\operatorname{Re}\left\{\mathbf{P}_{\Delta}\right\} & -\operatorname{Re}\left\{\mathbf{P}_{\nabla}\right\} & \mathbf{I} & 0 \\
-\operatorname{Im}\left\{\mathbf{P}_{\Delta}\right\} & -\operatorname{Im}\left\{\mathbf{P}_{\nabla}\right\} & 0 & \mathbf{I}
\end{array}\right]
$$

Thereafter, we can obtain $[\Delta, \nabla, \mathbf{H}, j \mathbf{H}] \mathbf{V}=\left[\left(\Delta-\mathbf{H P}_{\Delta}\right),(\nabla\right.$ $\left.\left.-\mathbf{H P}_{\nabla}\right), \mathbf{H}, \mathbf{j} \mathbf{H}\right]$. Let $\Pi_{\mathbf{H}}^{\perp}$ denote the orthogonal projection of $\mathbf{H}^{H}$ onto null space, i.e.,

$$
\Pi_{\mathbf{H}}^{\perp} \triangleq \mathbf{I}-\mathbf{H}\left(\mathbf{H}^{H} \mathbf{H}\right)^{-1} \mathbf{H}^{H}
$$

Notably, $\mathbf{H}^{H} \prod(1 / \mathbf{H})=0$. Then, the following result can be obtained:

$$
\begin{aligned}
\mathbf{V}^{H} \mathrm{JV} & =\operatorname{Rc}\left\{\left[\begin{array}{c}
\Delta^{H} \Pi_{\mathbf{H}}^{\perp} \\
\nabla^{H} \Pi_{\mathbf{H}}^{\perp} \\
\mathbf{H}^{H} \\
-j \mathbf{H}^{H}
\end{array}\right]\left[\Pi_{\mathbf{H}}^{\perp} \Delta, \Pi_{\mathbf{H}}^{\perp} \nabla, \mathbf{H}, j \mathbf{H}\right]\right\} \\
= & \operatorname{Re}\left\{\left[\begin{array}{cccc}
\Delta^{H} \Pi_{\mathbf{H}}^{\perp} \Delta & \Delta^{H} \Pi_{\mathbf{H}}^{\perp} \nabla & 0 & 0 \\
\nabla^{H} \Pi_{\mathbf{H}}^{\perp} \Delta & \nabla^{H} \Pi_{\mathbf{H}}^{\perp} \nabla & 0 & 0 \\
0 & 0 & \mathbf{H}^{H} \mathbf{H} & j \mathbf{H}^{H} \mathbf{H} \\
0 & 0 & -j \mathbf{H}^{H} \mathbf{H} & \mathbf{H}^{H} \mathbf{H}
\end{array}\right]\right\} .
\end{aligned}
$$
get

Applying the property of a partitioned diagonal matrix, we

$$
\mathbf{J}^{-1}=\mathbf{V}\left(\mathbf{V}^{H} \mathbf{J V}\right)^{-1} \mathbf{V}^{H}=\left[\begin{array}{ccc}
\operatorname{Re}\left\{\Delta^{H} \Pi_{\mathrm{H}}^{\perp} \Delta\right\} & \operatorname{Re}\left\{\Delta^{H} \Pi_{\mathrm{H}}^{\perp} \nabla\right\} & \oslash \\
\operatorname{Re}\left\{\nabla^{H} \Pi_{\mathrm{H}}^{\perp} \Delta\right\} & \operatorname{Re}\left\{\nabla^{H} \Pi_{\mathrm{H}}^{\perp} \nabla\right\} & \oslash \\
\oslash & \oslash & \oslash
\end{array}\right]^{-1},
$$

where $\oslash$ denotes the uninteresting term. As a result, the CRB matrix concerning angle and $\mathrm{MC}$ estimation is given by

$$
\mathrm{CRB}_{a, c}=\frac{\sigma^{2}}{2}\left[\begin{array}{ll}
\operatorname{Re}\left\{\Delta^{H} \Pi_{\mathbf{H}}^{\perp} \Delta\right\} & \operatorname{Re}\left\{\Delta^{H} \Pi_{\mathbf{H}}^{\perp} \nabla\right\} \\
\operatorname{Re}\left\{\nabla^{H} \Pi_{\mathbf{H}}^{\perp} \Delta\right\} & \operatorname{Re}\left\{\nabla^{H} \Pi_{\mathbf{H}}^{\perp} \nabla\right\}
\end{array}\right]^{-1} .
$$

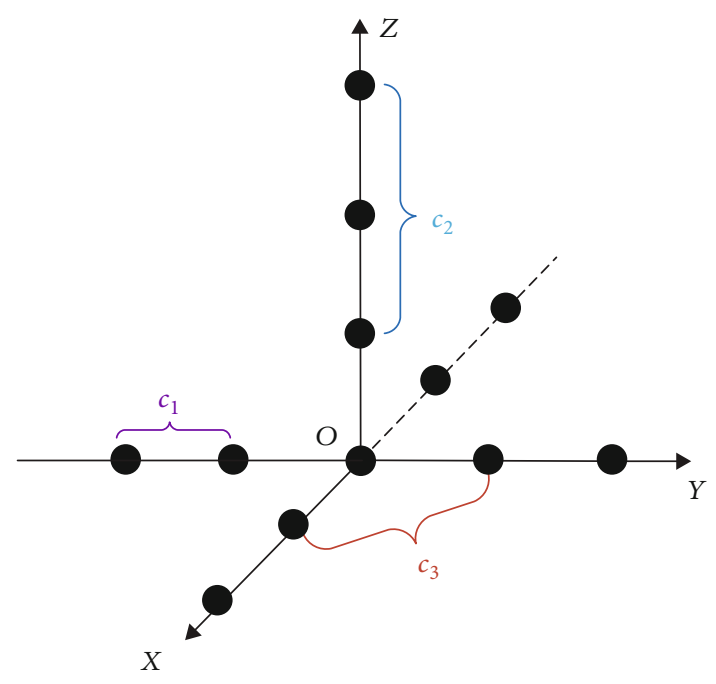

Figure 1: Illustration of 3D array.

According to the inverse property of a partitioned matrix, the $\mathrm{CRB}$ with respect to angle estimation $\mathrm{CRB}_{a}$ and MC estimation $\mathrm{CRB}_{c}$ are separately given by

$$
\begin{aligned}
\mathrm{CRB}_{a}= & {\left[\operatorname{Re}\left\{\Delta^{H} \Pi_{\mathbf{H}}^{\perp} \Delta\right\}-\operatorname{Re}\left\{\Delta^{H} \Pi_{\mathbf{H}}^{\perp} \nabla\right\} \operatorname{Re}^{-1}\right.} \\
& \left.\cdot\left\{\nabla^{H} \Pi_{\mathbf{H}}^{\perp} \nabla\right\} \operatorname{Re}\left\{\nabla^{H} \Pi_{\mathbf{H}}^{\perp} \Delta\right\}\right]^{-1}, \\
\mathrm{CRB}_{c}= & {\left[\operatorname{Re}\left\{\nabla^{H} \Pi_{\mathbf{H}}^{\perp} \nabla\right\}-\operatorname{Re}\left\{\nabla^{H} \Pi_{\mathbf{H}}^{\perp} \Delta\right\} \operatorname{Re}^{-1}\right.} \\
& \left.\cdot\left\{\Delta^{H} \Pi_{\mathbf{H}}^{\perp} \Delta\right\} \operatorname{Re}\left\{\Delta^{H} \Pi_{\mathbf{H}}^{\perp} \nabla\right\}\right]^{-1} .
\end{aligned}
$$

However, it may be impractical to calculate $\mathrm{CRB}_{a}$ and $\mathrm{CRB}_{c}$ via (41a) and (41b) due to the high-dimensional characteristics of the matrices $\Delta, \nabla$, and $\mathbf{H}$. Taking $\Delta^{H} \Pi_{\mathbf{H} \nabla}^{\perp}$ as an example, we will show a "faster" way to calculate $\mathrm{CRB}_{a}$ and $\mathrm{CRB}_{c}$. Note that $\mathbf{H}=I \otimes A$; hence,

$$
\Pi_{\mathbf{H}}^{\perp}=\mathbf{I} \otimes \Pi_{\mathbf{A}}^{\perp}
$$

On the other hand, both $\Delta$ and $\nabla$ can be divided into several blocks, as illustrated in (31) and (32), respectively. Let $\tilde{a}_{u}$ and $\tilde{b}_{v}$ be the $u$-th $(u \in\{1,2, \cdots, 4 K\})$ and $v$-th $(v \in\{1,2, \cdots, 2(P$ $+Q)\})$ columns of $\tilde{A}$ and $\tilde{B}$, respectively. Similarly, let $\mathbf{s}_{u}, \Delta_{u}$ and $\mathbf{s}_{v}, \nabla_{v}$ denote the $u$-th column and the $v$-th column of $1_{1 \times 4} \otimes \mathrm{S}, 1_{1 \times 2(\mathrm{P}+\mathrm{Q})} \otimes \mathrm{S}$ and $\Delta$ and $\nabla$, respectively. Recall the property $(A \otimes B)(C \otimes D)=(A C) \otimes(B D)$ yielding

$$
\begin{aligned}
\Delta_{u}^{H} \Pi_{\mathbf{H}}^{\perp} \nabla_{v} & =\left(\mathbf{s}_{u}^{H} \otimes \tilde{\mathbf{a}}_{u}^{H}\right)\left(\mathbf{I} \otimes \Pi_{\mathbf{A}}^{\perp}\right)\left(\mathbf{s}_{v} \otimes \tilde{\mathbf{b}}_{v}\right) \\
& =\left(\mathbf{s}_{u}^{H} \mathbf{s}_{v}\right) \otimes\left(\tilde{\mathbf{a}}_{u}^{H} \Pi_{\mathbf{A}}^{\perp} \tilde{\mathbf{b}}_{u}\right)=\mathbf{R}_{u, v} \cdot\left(\tilde{\mathbf{a}}_{u}^{H} \Pi_{\mathbf{A}}^{\perp} \tilde{\mathbf{b}}_{u}\right),
\end{aligned}
$$

where $\mathbf{R}_{u, v}$ is the $(u, v)$-th entity of the extended covariance matrix $1_{4 \times 2(P+Q)} \otimes \mathbf{R}_{s}$. Based on (43), we have

$$
\mathscr{M}_{2}=\Delta^{H} \Pi_{\mathbf{H}}^{\perp} \nabla=\left(\tilde{\mathbf{A}}^{H} \Pi_{A}^{\perp} \tilde{\mathbf{B}}\right) \oplus\left(1_{4 \times 2(P+Q)} \otimes \mathbf{R}_{s}\right) .
$$




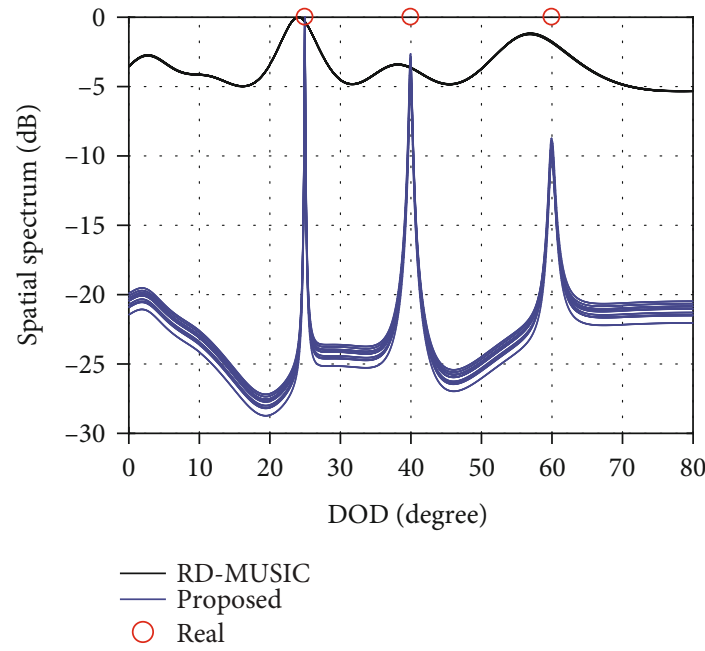

(a)

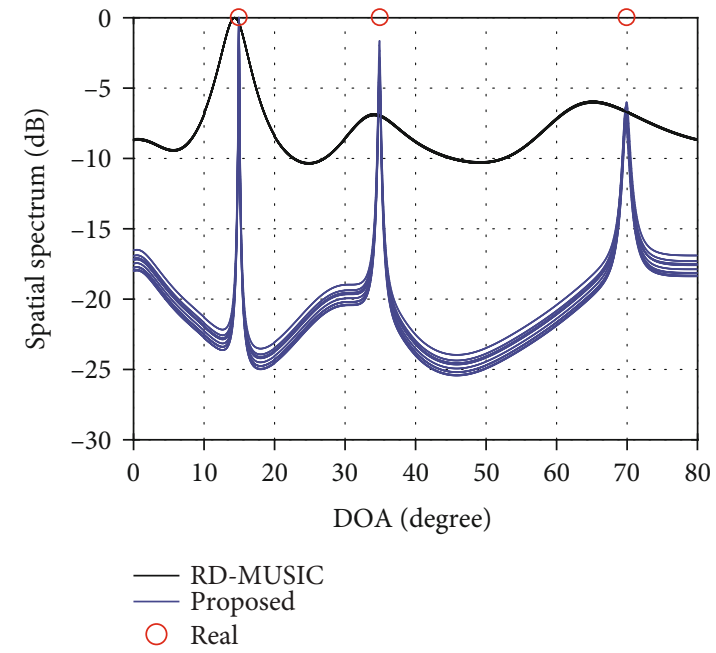

(b)

FIGURE 2: Spatial spectrum of the proposed estimator in Scenario I: (a) spatial spectrums of DOD estimation; (b) spectrum results of DOA estimation.

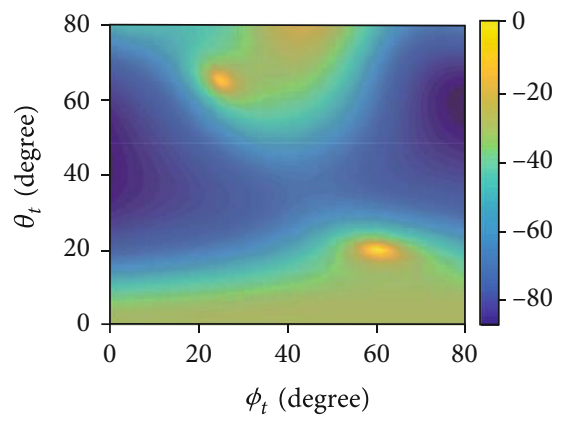

(a)

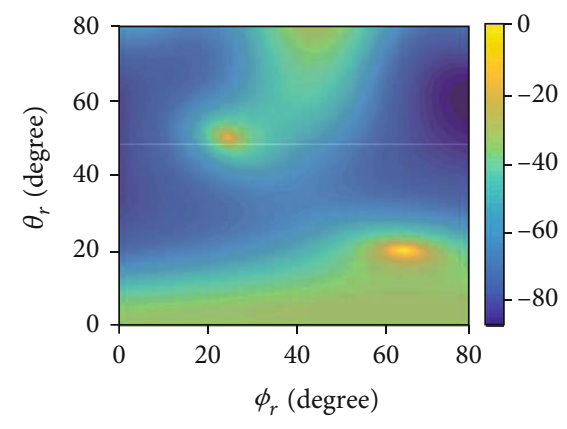

(b)

FIGURE 3: Spatial spectrum of the proposed estimator in Scenario II: (a) spatial spectrum of 2D-DOD estimation; (b) spectrum result of 2DDOA estimation.

Likewise, we define

$$
\begin{aligned}
& \mathscr{M}_{1}=\Delta^{H} \Pi_{\mathbf{H}}^{\perp} \Delta=\left(\tilde{\mathbf{A}}^{H} \Pi_{\mathbf{A}}^{\perp} \tilde{\mathbf{A}}\right) \oplus\left(1_{4 \times 4} \otimes \mathbf{R}_{s}\right), \\
& \mathscr{M}_{3}=\nabla^{H} \Pi_{\mathbf{H}}^{\perp} \Delta=\left(\tilde{\mathbf{B}}^{H} \Pi_{\mathbf{A}}^{\perp} \tilde{\mathbf{A}}\right) \oplus\left(1_{2(P+Q) \times 4} \otimes \mathbf{R}_{s}\right), \\
& \mathscr{M}_{4}=\nabla^{H} \Pi_{\mathbf{H}}^{\perp} \nabla=\left(\tilde{\mathbf{A}}^{H} \Pi_{\mathbf{B}}^{\perp} \tilde{\mathbf{B}}\right) \oplus\left(\mathbf{1}_{2(P+Q) \times 2(P+Q)} \otimes \mathbf{R}_{s}\right) .
\end{aligned}
$$
yields

Inserting (44), (45a), (45b), and (45c) into (41a) and (41b)

$$
\begin{aligned}
& \mathrm{CRB}_{a}=\left(\mathscr{M}_{1}-\mathscr{M}_{2} \mathscr{M}_{4}^{-1} \mathscr{M}_{3}\right)^{-1}, \\
& \mathrm{CRB}_{c}\left(\mathscr{M}_{4}-\mathscr{M}_{3} \mathscr{M}_{1}^{-1} \mathscr{M}_{2}\right)^{-1} .
\end{aligned}
$$

\section{Simulation Results}

In this section, numerical simulations are carried out to show the effectiveness of the proposed estimator. Specifically, we assume that the MIMO system is configured with $M$ transmit sensors and $N$ receive sensors. Suppose that there are $K$ targets, and each RCS fulfills the Swerling II model and $L=500$ snapshots are collected. Search interval in the simulation is set to $\Delta$. The following two scenarios are considered.

Scenario I. A ULA-based MIMO system with sensor failure, where $M=12$ and $N=12$, is where both the transmitters and the receivers are ULAs with the adjacent sensor distance $\lambda / 2$. There are two damaged transmit sensors with locations $\{7,10\}$, and two damaged receive sensors with locations $\{6$ $, 11\}$. The MC coefficient between two adjacent sensors is $0.8+0.5 j$, the MC coefficient of sensors with distance $\lambda$ is $0.2+0.1 j$, and there is no MC effect when the distance of sensors is larger than $\lambda$, i.e., $c_{t}=c_{r}=[1,0.8+0.5 j, 0.2+0.1 j]^{\mathrm{T}}$ Suppose that $K=3$ targets with DOD-DOA pairs are $\left(25^{\circ}\right.$, $\left.15^{\circ}\right)$ and $\left(60^{\circ}, 70^{\circ}\right)$. 


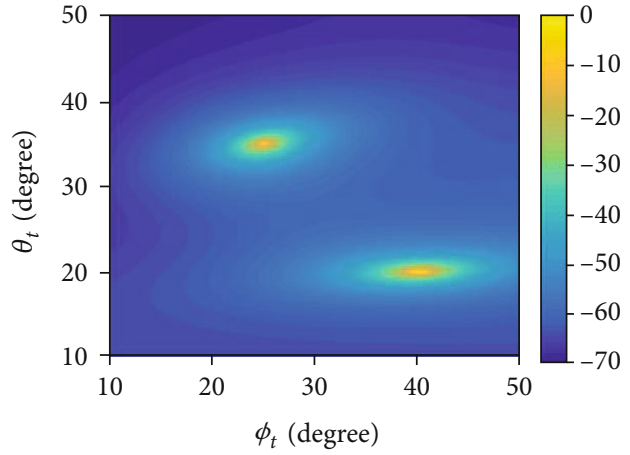

(a)

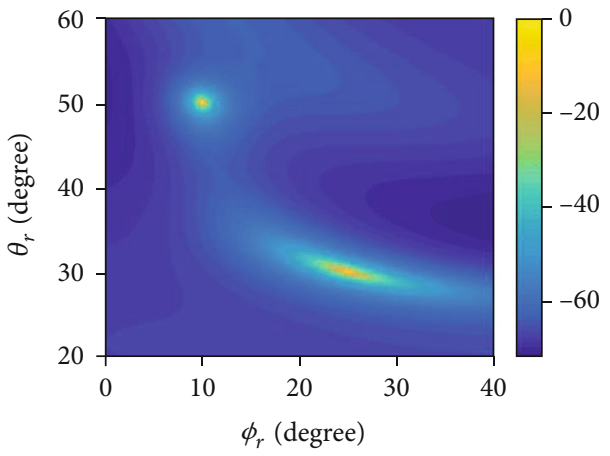

(b)

FIGURE 4: Spatial spectrum of the proposed estimator in Scenario II: (a) spatial spectrum of 2D-DOD estimation; (b) spectrum result of 2DDOA estimation.

Scenario II. A 3D-ULA-based MIMO system, where $M=N$ $=12$, wherein the adjacent sensor distance is $\lambda / 2$, as illustrated in Figure 1. The MC coefficient between two adjacent sensors is $0.8+0.5 j$, the MC coefficient of sensors with distance $\lambda$ is $0.2+0.1 j$, the MC coefficient between two crossadjacent sensors is $0.017+0.035 j$, and there is no MC effect when the distance of sensors is larger than $\lambda$, e.g., $c_{t}=c_{r}=$ $[1,0.8+0.5 j, 0.2+0.1 j, 0.017+0.035 j]^{\mathrm{T}}$. There are $K=2$ targets with transmit DOD-DOA pairs $\left(60^{\circ}, 70^{\circ}\right)$ and $\left(25^{\circ}\right.$, $\left.15^{\circ}\right)$ and receive DOD-DOA pairs $\left(65^{\circ}, 20^{\circ}\right)$ and $\left(25^{\circ}, 20^{\circ}\right)$.

Scenario III. Arbitrary Tx/Rx geometries. We assume $M=8$, $N=12$, and the $\mathrm{Tx} / \mathrm{Rx}$ sensors are randomly placed in a three-dimensional space with $\left(x_{t, m}, y_{t, m}, z_{t, m}\right) \sim \operatorname{unif}([-\lambda, \lambda])$ and $\left(x_{r, n}, y_{r, n}, z_{r, n}\right) \sim \operatorname{unif}([-2 \lambda, 2 \lambda]), L=500, \mathrm{SNR}=20 \mathrm{~dB}$, and search interval is fixed at $0.5^{\circ}$. There are $K=2$ far-field targets, the DOD pairs are $\left(20^{\circ}, 40^{\circ}\right)$ and $\left(35^{\circ}, 25^{\circ}\right)$, and the DOA pairs are $\left(50^{\circ}, 10^{\circ}\right)$ and $\left(30^{\circ}, 25^{\circ}\right)$. Moreover, $c_{t}=c_{r}=$ $[1,0.8+0.5 j, 0.017+0.035 j, 0.2+0.1 j]^{\mathrm{T}}$.

In the first example, we test the spatial spectrum of the proposed estimator in Scenario I, where $\Delta=0.01^{\circ}$, signalto-noise ratio (SNR) is set to $15 \mathrm{~dB}$, and 10 independent trials are recorded. The results are presented in Figure 2, from which we observe that the proposed estimator can accurately identify all the targets. For comparison purposes, the spectrum results of the traditional reduced-dimension MUSIC (RD-MUSIC) algorithm in [7] are added, which display great disparities for some angles. This is caused by the fact that the traditional RD-MUSIC cannot eliminate the MC effect.

In the second example, we give the $2 \mathrm{D}$ spectrum results of the proposed estimator in Scenario II, where $\Delta=0.01^{\circ}$ and $\mathrm{SNR}=15 \mathrm{~dB}$ are considered. Figure 3 illustrates the $2 \mathrm{D}$ spectrum results of the proposed estimator. Clearly, the proposed estimator can correctly recover the $2 \mathrm{D}$-DODs and the $2 \mathrm{D}$ DOAs, which is evident that the proposed estimator is also suitable for the MIMO system with MC and 3D sensor geometry.

In the third example, we plot the 2D spectrum results of the proposed estimator in Scenario III. Figure 4 gives the results. Similar to the previous simulation, the proposed esti-

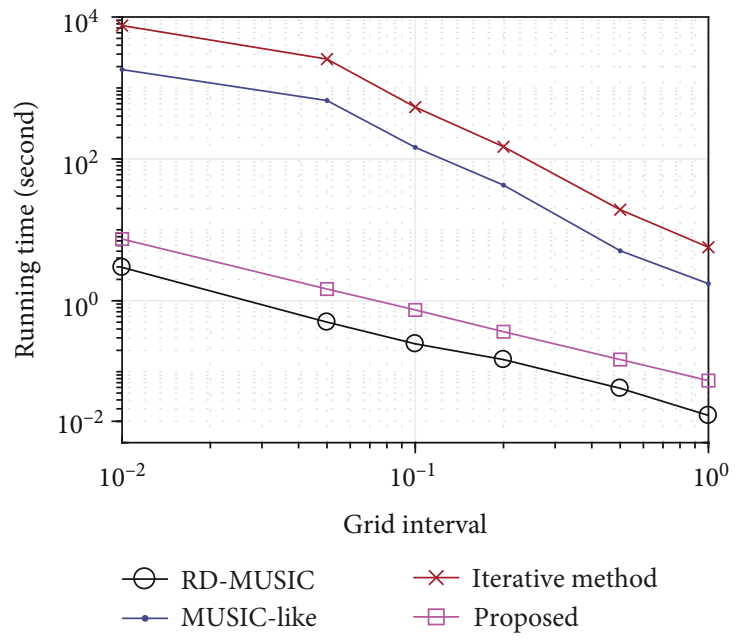

Figure 5: Average running time versus $\Delta$ in Scenario I.

mator can correctly estimate the 2D-DODs and the 2DDOAs in such a condition.

In the fourth example, we examine the average running time of the proposed estimator against different search interval $\Delta$ in Scenario I, where SNR is fixed at $20 \mathrm{~dB}$. The result is displayed in Figure 5. As expected, a refined search interval would result in more computational burden. For comparison purposes, the performances associated with the reduceddimension MUSIC in [32] (marked with "RD-MUSIC") and the iterative method in [33] (marked with "Iterative method") as well as the MUSIC-like method in [34] (marked with "MUSIC-like") are added. In contrast with RD-MUSIC, the proposed estimator requires a slightly more running time. However, it is shown that the running time of the proposed estimator is two orders of magnitude lower than that of the Iterative method and MUSIC-like. This is because both RD-MUSIC and the proposed estimator require two 1D spectrum searches, while the $2 \mathrm{D}$ spectrum search is essential in both the Iterative method and MUSIC-like.

Finally, we plot the average root mean square error (RMSE) curves of the proposed estimator versus SNR in Scenario I, where $\Delta=0.01^{\circ}$ and 200 Monte Carlo trials are carried out. As shown in Figure 6, the proposed estimator 


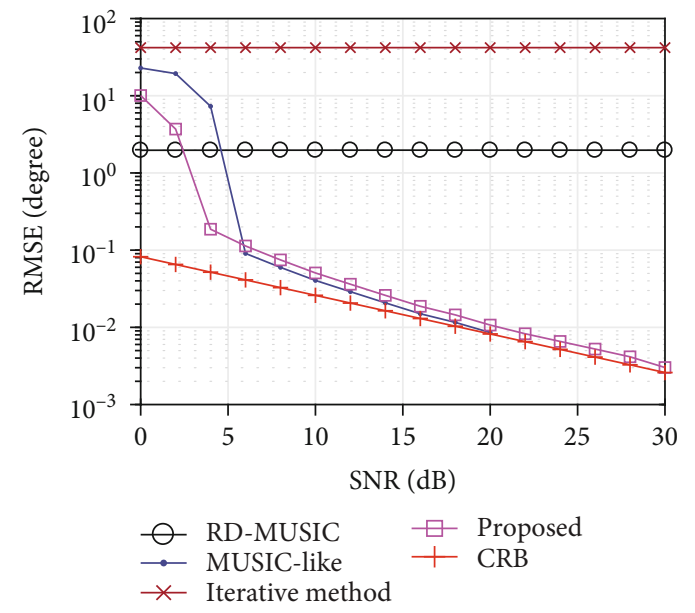

(a)

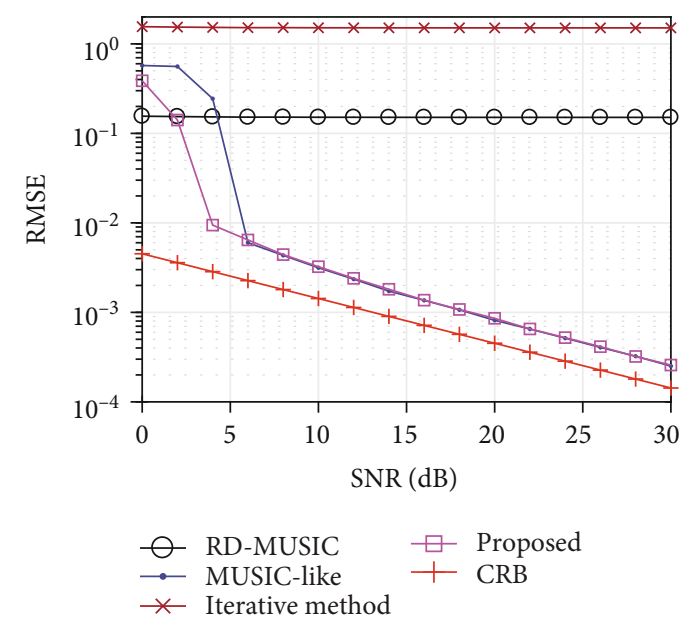

(b)

FIGURE 6: RMSE performance versus $L$ in Scenario I: (a) RMSE on angle estimation; (b) RMSE on MC coefficient estimation.

deteriorates in the low SNR regime (e.g., SNR $<4 \mathrm{~dB}$ ). With the increasing SNR, the proposed estimator would achieve improved RMSE performance, and it may attain the CRB at high SNR regions. An interesting observation is that the proposed estimator offers better performance than MUSIC-like when SNR is smaller than $6 \mathrm{~dB}$, and it provides very close RMSE performance to the latter when SNR is larger than $6 \mathrm{~dB}$, especially in MC estimation. Nevertheless, both RDMUSIC and Iterative method provide the unacceptable RMSE performance over the entire SNR regions.

\section{Conclusion}

In this paper, we presented a MUSIC-like estimator for bistatic MIMO radar with arbitrary geometries and MC. For the 1D sensor manifold scenario, the proposed estimator only needs two one-dimensional spectrum peak searches to obtain 1D-DODs and 1D-DOAs, while for 2D or 3D sensor geometries, the proposed estimator allows two 2D spectrum searches to achieve 2D-DODs and 2D-DOAs. The proposed estimator is efficient from the viewpoint of complexity. It should have a brighter prospect in numerous applications, e.g., military explorations and internet-of-vehicles.

\section{Data Availability}

MATLAB codes are available on "https://pan.baidu.com/s/ 1y8WMKJa2s6MMpf5Vu2KFTg” with security code "rbii."

\section{Conflicts of Interest}

The authors declare no conflict of interest.

\section{Acknowledgments}

This work was supported by the Ministry of Education of Humanities and Social Science Project under grant 20YJAZH132 and Natural Science Foundation Project of
CQCST under grant cstc2018jcyjAX0398. Also, it is supported by China NSF grants (61701046).

\section{References}

[1] E. Fishler, A. Haimovich, R. Blum, D. Chizhik, L. Cimini, and R. Valenzuela, "MIMO radar: an idea whose time has come," in Proceedings of the 2004 IEEE Radar Conference (IEEE Cat. No.04CH37509), pp. 71-78, Philadelphia, PA, USA, 2004.

[2] L. Wan, L. Sun, K. Liu, X. Wang, Q. Lin, and T. Zhu, "Autonomous vehicle source enumeration exploiting non-cooperative UAV in software defined internet of vehicles," IEEE Transactions on Intelligent Transportation Systems, vol. 22, no. 6, pp. 3603-3615, 2021.

[3] J. Li, Y. He, X. Zhang, and Q. Wu, "Simultaneous localization of multiple unknown emitters based on UAV monitoring big data," IEEE Transactions on Industrial Informatics, vol. 17, no. 9, pp. 6303-6313, 2021.

[4] A. M. Haimovich, R. S. Blum, and L. J. Cimini, "MIMO radar with widely separated antennas," IEEE Signal Processing Magazine, vol. 25, no. 1, pp. 116-129, 2008.

[5] J. Li and P. Stoica, "MIMO radar with colocated antennas," IEEE Signal Processing Magazine, vol. 24, no. 5, pp. 106-114, 2007.

[6] C. Jinli, G. Hong, and S. Weimin, "Angle estimation using ESPRIT without pairing in MIMO radar," Electronics Letters, vol. 44, no. 24, pp. 1422-1423, 2008.

[7] X. Zhang, L. Xu, L. Xu, and D. Xu, "Direction of departure (DOD) and direction of arrival (DOA) estimation in MIMO radar with reduced-dimension MUSIC," IEEE Communications Letters, vol. 14, no. 12, pp. 1161-1163, 2010.

[8] B. Tang, J. Tang, Y. Zhang, and Z. Zheng, "Maximum likelihood estimation of DOD and DOA for bistatic MIMO radar," Signal Processing, vol. 93, no. 5, pp. 1349-1357, 2013.

[9] X. Zhang, Z. Xu, L. Xu, and D. Xu, "Trilinear decompositionbased transmit angle and receive angle estimation for multipleinput multiple-output radar," IET Radar Sonar ? Navigation, vol. 5, no. 6, pp. 626-631, 2011.

[10] Y. Cheng, R. Yu, H. Gu, and W. Su, "Multi-SVD based subspace estimation to improve angle estimation accuracy in 
bistatic MIMO radar," Signal Processing, vol. 93, no. 7, pp. 2003-2009, 2013.

[11] X. Wang, L. Wang, X. Li, and G. Bi, "Nuclear norm minimization framework for DOA estimation in MIMO radar," Signal Processing, vol. 135, pp. 147-152, 2017.

[12] X. Wang, L. T. Yang, D. Meng, M. Dong, K. Ota, and H. Wang, "Multi-UAV cooperative localization for marine targets based on weighted subspace fitting in SAGIN environment," IEEE Internet of Things Journal, 2021.

[13] E. Boudaher, F. Ahmad, M. G. Amin, and A. Hoorfar, "Mutual coupling effect and compensation in non-uniform arrays for direction-of-arrival estimation," Digital Signal Processing, vol. 61, pp. 3-14, 2017.

[14] T. Katagi, H. Ohmine, H. Miyashita, and K. Nishimoto, "Analysis of mutual coupling between dipole antennas using simultaneous integral equations with exact kernels and finite gap feeds," IEEE Transactions on Antennas and Propagation, vol. 64, no. 5, pp. 1979-1984, 2016.

[15] M. Wang, Z. Wang, and Z. Cheng, "Joint calibration of mutual coupling and channel gain/phase inconsistency using a nearfield auxiliary source," in 2016 IEEE 13th International Conference on Signal Processing (ICSP), pp. 394-398, Chengdu, China, 2016.

[16] Y. Guo, Y. Zhang, N. Tong, and J. Gong, “Angle estimation and self-calibration method for bistatic MIMO radar with transmit and receive array errors," Circuits, Systems, and Signal Processing, vol. 36, no. 4, pp. 1514-1534, 2017.

[17] J. Li, D. Jiang, and X. Zhang, "DOA estimation based on combined unitary ESPRIT for coprime MIMO radar," IEEE Communications Letters, vol. 21, no. 1, pp. 96-99, 2017.

[18] J. Shi, F. Wen, and T. Liu, "Nested MIMO radar: coarrays, tensor modeling, and angle estimation," IEEE Transactions on Aerospace and Electronic Systems, vol. 57, no. 1, pp. 573-585, 2021.

[19] X. Liu and G. Liao, "Direction finding and mutual coupling estimation for bistatic MIMO radar," Signal Processing, vol. 92, no. 2, pp. 517-522, 2012.

[20] Z. Zheng, J. Zhang, and J. Zhang, "Joint DOD and DOA estimation of bistatic MIMO radar in the presence of unknown mutual coupling," Signal Processing, vol. 92, no. 12, pp. 3039-3048, 2012.

[21] J. Li and X. Zhang, "Joint angles and mutual coupling estimation algorithm for bistatic MIMO radar," International Journal of Antennas and Propagation, vol. 2012, no. 8, Article ID 921878, p. 8, 2012.

[22] X. Wang, W. Wang, J. Liu, Q. Liu, and B. Wang, "Tensorbased real-valued subspace approach for angle estimation in bistatic MIMO radar with unknown mutual coupling," Signal Process, vol. 116, no. 292, pp. 152-158, 2015.

[23] F. Wen, X. Xiong, and Z. Zhang, "Angle and mutual coupling estimation in bistatic MIMO radar based on PARAFAC decomposition," Digital Signal Processing, vol. 65, pp. 1-10, 2017.

[24] F. Wen, Z. Zhang, K. Wang, G. Sheng, and G. Zhang, "Angle estimation and mutual coupling self-calibration for ULAbased bistatic MIMO radar," Signal Processing, vol. 144, pp. 61-67, 2018.

[25] F. Wen, J. Shi, and Z. Zhang, "Closed-form estimation algorithm for EMVS-MIMO radar with arbitrary sensor geometry," Signal Processing, vol. 186, article 108117, 2021.
[26] X. Wang, M. Huang, and L. Wan, "Joint 2D-DOD and 2DDOA estimation for coprime EMVS-MIMO radar," Circuits, Systems, and Signal Processing, vol. 40, no. 6, article 1605, pp. 2950-2966, 2021.

[27] J. He, L. Li, and T. Shu, "Sparse nested arrays with spatially spread orthogonal dipoles: high accuracy passive direction finding with less mutual coupling," IEEE Transactions on Aerospace and Electronic Systems, p. 1, 2021.

[28] L. Wan, K. Liu, Y.-C. Liang, and T. Zhu, "DOA and polarization estimation for non-circular signals in 3-D millimeter wave polarized massive MIMO systems," IEEE Wireless Communications, vol. 20, no. 5, pp. 3152-3167, 2021.

[29] J. Chen, T. Zhang, J. Li, and X. Chen, "Joint sensor failure detection and corrupted covariance matrix recovery in bistatic MIMO radar with impaired arrays," IEEE Sensors Journal, vol. 19, no. 14, pp. 5834-5842, 2019.

[30] J. Chen, Q. Zhuo, J. Li, and Y. Zhu, “Array diagnosis and angle estimation in bistatic MIMO radar under array antenna failures," IET Radar, Sonar \& Navigation, vol. 13, no. 7, pp. 1180-1188, 2019.

[31] T. Xia, "Joint diagonalization based 2D-DOD and 2D-DOA estimation for bistatic MIMO radar," Signal Processing, vol. 116, pp. 7-12, 2015.

[32] M. Ahmet and T. T. Engin, "2-d DOA and mutual coupling coefficient estimation for arbitrary array structures with single and multiple snapshots," Digital Signal Process, vol. 54, pp. 7586, 2016.

[33] A. M. Elbir, "A novel data transformation approach for DOA estimation with 3-D antenna arrays in the presence of mutual coupling," IEEE Antennas and Wireless Propagation Letters, vol. 16, pp. 2118-2121, 2017.

[34] F. Wen, J. Wang, J. Shi, and G. Gui, “Auxiliary vehicle positioning based on robust DOA estimation with unknown mutual coupling," IEEE Internet of Things Journal, vol. 7, no. 6, pp. 5521-5532, 2020. 